\title{
The expression and clinical value of tumor infiltrating dendritic cells in tumor tissues of patients with esophageal cancer
}

\author{
Yanzhi Pei ${ }^{1 \#}$, Yanzhi Zhu ${ }^{2 \#}$, Xiaolin Wang ${ }^{3 \#}$, Lin $\mathrm{Xu}^{4}$ \\ ${ }^{1}$ Department of Thoracic Surgery, First Affiliated Hospital of Jiamusi University, Jiamusi, China; ${ }^{2}$ Hepatobiliary and Pancreatic Surgery, Taihe \\ Hospital Affiliated to Hubei University of Medicine, Shiyan, China; ${ }^{3}$ Department of Pathology, Weifang Traditional Chinese Hospital, Weifang, \\ China; ${ }^{4}$ Department of Gastroenterology, Xuzhou Cancer Hospital, Xuzhou, China \\ Contributions: (I) Conception and design: Y Pei, L Xu; (II) Administrative support: Y Pei, L Xu; (III) Provision of study materials or patients: All \\ authors; (IV) Collection and assembly of data: All authors; (V) Data analysis and interpretation: All authors; (VI) Manuscript writing: All authors; (VII) \\ Final approval of manuscript: All authors. \\ \#These authors contributed equally to this work. \\ Correspondence to: Lin Xu. Department of Gastroenterology, Xuzhou Cancer Hospital, Xuzhou 221000, China. Email: xulin1635@sina.com.
}

Background: As dendritic cells (DCs) are the major antigen-presenting cells of the immune system, understanding their role in esophageal cancer is essential for the development of preventative and treatment strategies. This study investigated the expression level and clinical value of tumor infiltrating dendritic cells (TIDCs) in tumor tissues of patients with esophageal cancer.

Methods: From January 2019 to January 2021, 184 patients with esophageal cancer treated were prospectively enrolled as the observation group and 184 patients with benign esophageal tumors were selected as the control group. Tumor tissue samples were obtained and the expression level and phenotypes of the TIDCs were analyzed. The correlation between TIDC expression and clinical characteristics of patients with esophageal cancer was investigated.

Results: The density of the TIDCs in the observation group was lower than that in the control group $(8.76 \pm 2.25$ vs. $9.97 \pm 2.19 ; \mathrm{P}=0.000)$. Furthermore, the percentage of major histocompatibility complex-II (MHC-II) positive DCs and the percentage of CD54 positive DCs were relatively lower in the observation group compared to the control group $(6.60 \% \pm 2.12 \%$ vs. $9.34 \% \pm 2.41 \% ; \mathrm{P}=0.000$ and $7.41 \% \pm 2.36 \%$ vs. $9.98 \% \pm 2.47 \% ; \mathrm{P}=0.000$, respectively). Esophageal cancer patients with lymph node metastasis had lower TIDC density, lower percentage of MHC-II positive DCs, and lower percentage of CD54 positive DCs compared to patients without node metastasis $(\mathrm{P}<0.05)$. Patients with stage III esophageal cancer also showed significantly lower TIDC density, lower percentage of MHC-II positive DCs, and lower percentage of CD54 positive DCs compared to patients with stage I/II esophageal cancer $(\mathrm{P}<0.05)$. Esophageal cancer patients with tumor diameter $\geq 4 \mathrm{~cm}$ presented with decreased TIDC density, decreased percentage of MHCII positive DCs, and decreased percentage of CD54 positive DCs compared to patients with tumor diameter $<4 \mathrm{~cm}(\mathrm{P}<0.05)$. In addition, the density of TIDCs, the percentage of MHC-II positive DCs, and the percentage of CD54 positive DCs were significantly negatively correlated with the percentage of CD4 ${ }^{+}$ T-lymphocytes and positively correlated with the percentage of $\mathrm{CD}^{+} \mathrm{T}$-lymphocytes $(\mathrm{P}<0.05)$.

Conclusions: Patients with esophageal cancer had low expression and function of TIDCs, and this was related to the imbalance of T-lymphocyte subsets, lymph node metastasis, TNM stage, and lesion size.

Keywords: Esophageal cancer; tumor infiltrating dendritic cells (TIDCs); T-lymphocyte subsets; phenotypes

Submitted Aug 19, 2021. Accepted for publication Sep 28, 2021.

doi: 10.21037/jgo-21-578

View this article at: https://dx.doi.org/10.21037/jgo-21-578

(C) Journal of Gastrointestinal Oncology. All rights reserved. 


\section{Introduction}

Esophageal cancer is a common malignant tumor of the digestive system. It can metastasize in the early stages and is associated with high postoperative recurrence and mortality (1-3). The metastasis of tumor cells is closely related to the biological characteristics of the tumor. Some esophageal cancer cells have strong invasiveness and infiltration ability. The immune system plays a key role in the occurrence and development of malignant tumors. Studies have shown that there is an imbalance of T-lymphocyte subsets in a variety of malignant tumor tissues, which is manifested as a drift of helper T-lymphocyte 1 (Th1)/helper T-lymphocyte 2 (Th2) immunity to Th2 immunity (4), an increase in regulatory T-lymphocytes $(5,6)$, and a decrease in the number of cytotoxic T-lymphocytes $(7,8)$. This leads a reduced ability to kill tumor cells, resulting in the immune escape of tumor cells. Dendritic cells (DC) are the most powerful antigen-presenting cells. They can transmit the information of molecular markers and metabolites on the surface of tumor cells to T-lymphocytes, stimulate the proliferation and activation of T-lymphocytes, produce a large number of cytotoxic T-lymphocytes, thereby killing tumor cells. Tumor infiltrating dendritic cells (TIDCs) refer to DCs infiltrating tumor tissues and these can reflect the anti-tumor immune ability of the patient. Recent studies have shown that the expression of TIDCs is reduced in a variety of tumor tissues and these TIDCs are functionally immature (9-17), resulting in reduced ability to stimulate T-lymphocyte proliferation and activation. These results suggested that reduction in DC numbers may be one mechanisms by which abnormal quantity and function of T-lymphocyte subsets affect malignant tumors. DCs in esophageal tissue may play an important role in tumor immunity (18) and studies have shown that increases in the number of DCs can improve the immune function and prolong the survival time of patients with esophageal cancer $(19,20)$. DCs can kill tumor cells by stimulating the proliferation of $\mathrm{T}$ cells, and then inhibit the proliferation and metastasis of tumor cells. Thus, the expression of DCs in patients with esophageal cancer may play a crucial role in the prognosis of these patients. However, there is a paucity of data exploring the expression levels and its functions of TIDCs in tumor tissues of patients with esophageal cancer. Moreover, the association between the clinical characters and TIDCs in tumor tissues of patients with esophageal cancer was not clear. This study investigated the expression level and clinical value of TIDCs in tumor tissues of patients with esophageal cancer.

We present the following article in accordance with the MDAR reporting checklist (available at https://dx.doi. org/10.21037/jgo-21-578).

\section{Methods}

\section{General data}

A total of 184 patients with esophageal cancer treated in our hospital from January 2019 to January 2021 were enrolled in this study. The following inclusion criteria were applied: (I) patients aged 18-74 years old with a positive pathological diagnosis of esophageal cancer; (II) esophageal cancer tissue could be obtained; and (III) the patient provided signed informed consent. Patients were excluded if they presented with any of the following: (I) postoperative recurrence of esophageal cancer; (II) metastatic esophageal cancer; (III) other types of malignant tumors; (IV) liver or kidney dysfunction; (V) cardiac, cerebral, or pulmonary insufficiencies; (VI) special treatment such as radiotherapy, chemotherapy, or molecular biological therapy before the operation; (VII) use of immunomodulator within 1 month of the operation; (VIII) other immune diseases such as ulcerative colitis; or (IX) infections. According to the above criteria, 184 patients with esophageal cancer, including 126 males and 58 females, aged 38-75 years (mean age $59.62 \pm 5.12$ years) were enrolled in the observation group. There are 76 patients with lymph node metastasis, 80 patients with TNM stage I/II, and 104 patients with TNM stage III. All patients presented with squamous cell carcinoma. Low or middle grade differentiation of tumor cells was observed in 60 cases and high tumor cell differentiation was noted in 124 cases. The maximum diameter of the tumor was $1.78-7.86 \mathrm{~cm}$ (mean diameter $3.65 \pm 1.24 \mathrm{~cm}$ ). A total of 184 patients with benign esophageal masses treated in our hospital during the same period were selected as the control group. All procedures performed in this study involving human participants were in accordance with the Declaration of Helsinki (as revised in 2013). The study was approved by the ethics committee of the First Affiliated Hospital of Jiamusi University (Ethics Approval No.: 2019-01-23) and informed consent was taken from all the patients.

\section{Outcome measures}

The percentage of TIDCs, major histocompatibility 
Table 1 The expression and phenotypic changes of TIDCs

\begin{tabular}{lcccc}
\hline Group & $\mathrm{N}$ & Density of TIDCs & MHC-II positive DCs (\%) & CD54 positive DCs (\%) \\
\hline Observation & 184 & $8.76 \pm 2.25$ & $6.60 \pm 2.12$ & $7.41 \pm 2.36$ \\
Control & 184 & $9.97 \pm 2.19$ & $9.34 \pm 2.41$ & $9.98 \pm 2.47$ \\
t value & & 5.227 & 11.579 & 10.205 \\
$P$ value & & 0.000 & 0.000 & 0.000 \\
\hline
\end{tabular}

TIDCs, tumor infiltrating dendritic cells; MHC-II, major histocompatibility complex-II; DCs, dendritic cells.

complex-II (MHC-II) positive DCs, and CD54 positive DCs in the lesion tissues of the patients were assessed. The percentage of $\mathrm{CD}^{+} \mathrm{T}$-lymphocytes and $\mathrm{CD} 8^{+}$ T-lymphocytes in the lesion tissues was also examined. The clinical characteristics of the patients, including age, gender, lymph node metastasis, TNM stage, tumor diameter, and tumor cell differentiation, were collated.

\section{Detection methods}

The percentage of $\mathrm{CD}^{+} \mathrm{T}$-lymphocytes and $\mathrm{CD} 8^{+}$ T-lymphocytes in the esophageal cancer tissues and benign esophageal tumor tissues were assessed by flow cytometry (Beckman Coulter, Epics XL, USA).

To determine the percentage of TIDCs, MHC-II positive DCs, and CD54 positive DCs in the lesion tissues, tumor tissues were obtained more than $0.8 \mathrm{~cm}$ from the tumor edge of esophageal cancer patients. In the control group, benign tumor tissues were harvested. The lesion tissues were stained by immunohistochemistry. Five visual fields were randomly chosen for observation under a microscope at 200x magnification. The number of TIDCs was counted and the mean value was calculated. MHCII and CD54 phenotypes of DCs were detected by flow cytometry (Beckman Coulter, Epics XL).

\section{Statistical analysis}

All statistical analyses were performed using the SPSS 22.0 software. An $\alpha$ value of 0.05 was set as the inspection standard. All tests were bilateral tests. Measurement data is expressed as mean \pm standard deviation and the differences between the two groups were analyzed by independent sample $t$-test. Pearson linear correlation analysis was used to analyze the correlation between the measurement data.

\section{Results}

\section{The expression and phenotype changes of TIDCs}

The density of the TIDCs in samples from patients with esophageal cancer (the observation group) was lower than that in patients with benign esophageal masses (the control group) $(8.76 \pm 2.25$ vs. 9.97 $\pm 2.19 ; \mathrm{P}=0.000)$. Furthermore, the percentage of MHC-II positive DCs detected in the observation group was relatively lower compared to that observed in the control group $(6.60 \% \pm 2.12 \%$ vs. $9.34 \% \pm 2.41 \% ; \mathrm{P}=0.000$; Table 1$)$. Similarly, the percentage of CD54 positive DCs was relatively lower in the observation group compared to the control group $(7.41 \% \pm 2.36 \%$ vs. $9.98 \% \pm 2.47 \% ; \mathrm{P}=0.000$; Table 1 and Figure 1).

\section{The expression of T-lymphocyte subsets}

The expression of $\mathrm{CD} 4^{+} \mathrm{T}$-lymphocytes in the observation groups was higher than that in the control group ( $24.81 \% \pm 3.72 \%$ vs. $20.77 \% \pm 3.63 \% ; \mathrm{P}=0.000$; Table 2$)$. Conversely, the expression of $\mathrm{CD} 8^{+} \mathrm{T}$-lymphocytes was lower in the observation group compared to the control group $(20.90 \% \pm 4.12 \%$ vs. $23.08 \% \pm 4.42 \% ; \mathrm{P}=0.000$; Table 2).

\section{The correlation between the expression of TIDCs in esophageal cancer tissues and the clinical characteristics of patients with esophageal cancer}

In esophageal cancer patients with lymph node metastasis, the density of the TIDCs was lower than that observed in patients without lymph node metastasis $(\mathrm{P}<0.05)$. Furthermore, patients with TNM stage III carcinoma had lower TIDC density compared to patients with TNM 

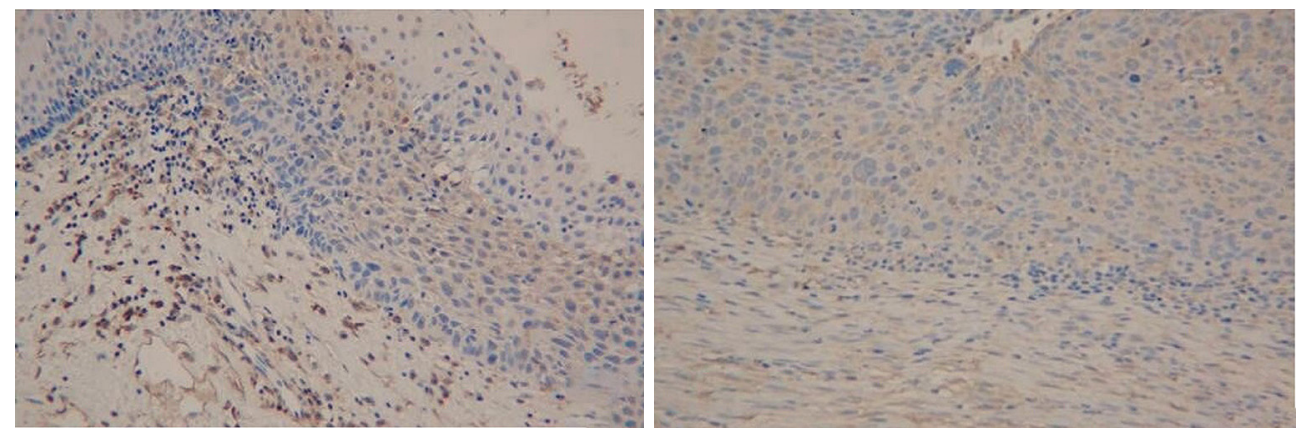

Figure 1 TIDCs showed dark-brown staining. A figure showed that TIDCs were mainly distributed in the peritumoral area but were sparsely present in the intratumoral area (immunohistochemical method, $\times 200$ ). TIDCs, tumor infiltrating dendritic cells.

Table 2 The expression of T-lymphocyte subsets

\begin{tabular}{lccc}
\hline Group & $\mathrm{N}$ & $\begin{array}{c}\mathrm{CD} 4^{+} \text {T-lymphocytes } \\
(\%)\end{array}$ & $\begin{array}{c}\mathrm{CD}^{+} \text {T-lymphocytes } \\
(\%)\end{array}$ \\
\hline Observation & 184 & $24.81 \pm 3.72$ & $20.90 \pm 4.12$ \\
Control & 184 & $20.77 \pm 3.63$ & $23.08 \pm 4.42$ \\
t value & & 10.544 & 4.894 \\
P value & & 0.000 & 0.000 \\
\hline
\end{tabular}

stage I/II esophageal cancer $(\mathrm{P}<0.05)$. Patients with tumor diameter $\geq 4 \mathrm{~cm}$ also showed lower TIDC density in the esophageal cancer tissues compared to patients with tumor diameter $<4 \mathrm{~cm}(\mathrm{P}<0.05$; Table 3$)$.

\section{The correlation between the expression of MHC-II positive DCs in esophageal cancer tissues and the clinical characteristics of patients with esophageal cancer}

The percentage of MHC-II positive DCs in esophageal cancer with lymph node metastasis was lower compared to patients without lymph node metastasis $(\mathrm{P}<0.05)$. Furthermore, patients with stage III esophageal cancer had proportionately fewer MHC-II positive DCs compared to patients with stage $\mathrm{I} / \mathrm{II}$ cancer $(\mathrm{P}<0.05)$. Patients with tumor diameter $\geq 4 \mathrm{~cm}$ also had fewer MHC-II positive DCs in the esophageal cancer tissues compared to patients with of patients with tumor diameter $<4 \mathrm{~cm}(\mathrm{P}<0.05$; Table 4$)$.

\section{The correlation between the expression of $\mathrm{CD} 54$ positive DCs in esophageal cancer tissues and the clinical characteristics of patients with esophageal cancer}

The percentage of CD54 positive DCs in esophageal cancer
Table 3 The correlation between the expression of TIDCs in esophageal cancer tissues and the clinical characteristics of patients with esophageal cancer

\begin{tabular}{lcccc}
\hline Category & $\mathrm{N}$ & TIDCs & t value & P value \\
\hline Age & & & 0.060 & 0.952 \\
$\geq 60$ years & 96 & $8.75 \pm 2.26$ & & \\
$<60$ years & 88 & $8.77 \pm 2.27$ & & \\
Gender & & & 0.250 & 0.803 \\
Male & 126 & $8.73 \pm 2.17$ & & \\
Female & 58 & $8.82 \pm 2.46$ & & \\
Lymphatic metastasis & & & 4.108 & 0.000 \\
Yes & 76 & $7.98 \pm 2.18$ & & \\
No & 108 & $9.31 \pm 2.15$ & & \\
TNM stage & & & 4.132 & 0.000 \\
Stage I/II & 80 & $9.51 \pm 2.27$ & & \\
Stage III & 104 & $8.18 \pm 2.08$ & & \\
Grade of differentiation & & & 0.930 & 0.354 \\
Middle or low & 60 & $8.98 \pm 2.23$ & & \\
High & 124 & $8.65 \pm 2.27$ & & \\
Tumor diameter & & & 4.979 & 0.000 \\
$\geq 4$ cm & 86 & $7.93 \pm 2.11$ & & \\
$<4$ cm & $9.49 \pm 2.13$ & & \\
\hline
\end{tabular}

TIDCs, tumor infiltrating dendritic cells.

patients with lymph node metastasis was lower compared to patients without lymph node metastasis $(\mathrm{P}<0.05)$. Patients with stage III esophageal cancer showed fewer CD54 positive DCs in esophageal cancer tissues compared to patients with 
Table 4 The correlation between the expression of MHCII positive DCs in esophageal cancer tissues and the clinical characteristics of patients with esophageal cancer

\begin{tabular}{|c|c|c|c|c|}
\hline Category & $\mathrm{N}$ & $\begin{array}{c}\text { MHC-II positive } \\
\text { DCs (\%) }\end{array}$ & $\mathrm{t}$ value & $P$ value \\
\hline Age & & & 0.127 & 0.899 \\
\hline$\geq 60$ years & 96 & $6.58 \pm 2.07$ & & \\
\hline$<60$ years & 88 & $6.62 \pm 2.20$ & & \\
\hline Gender & & & 0.857 & 0.392 \\
\hline Male & 126 & $6.51 \pm 2.08$ & & \\
\hline Female & 58 & $6.80 \pm 2.24$ & & \\
\hline Lymphatic metastasis & & & 8.023 & 0.000 \\
\hline Yes & 76 & $5.31 \pm 1.79$ & & \\
\hline No & 108 & $7.51 \pm 1.86$ & & \\
\hline TNM stage & & & 4.751 & 0.001 \\
\hline Stage $\mathrm{I} / \mathrm{II}$ & 80 & $7.40 \pm 2.01$ & & \\
\hline Stage III & 104 & $5.98 \pm 2.01$ & & \\
\hline Grade of differentiation & & & 1.381 & 0.169 \\
\hline Middle or low & 60 & $6.29 \pm 2.07$ & & \\
\hline High & 124 & $6.75 \pm 2.14$ & & \\
\hline Tumor diameter & & & 6.030 & 0.000 \\
\hline$\geq 4 \mathrm{~cm}$ & 86 & $5.68 \pm 2.01$ & & \\
\hline$<4 \mathrm{~cm}$ & 98 & $7.41 \pm 1.88$ & & \\
\hline
\end{tabular}

MHC-II, major histocompatibility complex-II; DC, dendritic cells.

TNM stage I/II $(\mathrm{P}<0.05)$. The percentage of $\mathrm{CD} 54$ positive DCs in esophageal cancer tissues of patients with tumor diameter $\geq 4 \mathrm{~cm}$ was also lower than that observed in patients with tumor diameter $<4 \mathrm{~cm}(\mathrm{P}<0.05$; Table 5$)$.

\section{The correlation of TIDC expression and phenotypes with T-lymphocyte subsets in esophageal cancer tissues}

The density of TIDCs, the percentage of MHC-II positive DCs, and the percentage of CD54 positive DCs were all negatively correlated with the percentage of $\mathrm{CD} 4{ }^{+}$T-lymphocytes and positively correlated with the percentage of $\mathrm{CD}^{+} \mathrm{T}$-lymphocytes $(\mathrm{P}<0.05$; Table 6).

\section{Discussion}

Tumor infiltrating immune cells play a key role in the
Table 5 The correlation between the expression of CD54 positive DCs in esophageal cancer tissues and the clinical characteristics of patients with esophageal cancer

\begin{tabular}{|c|c|c|c|c|}
\hline Category & $\mathrm{N}$ & $\begin{array}{l}\text { CD54 positive } \\
\text { DCs (\%) }\end{array}$ & $\mathrm{t}$ value & $P$ value \\
\hline Age & & & 0.486 & 0.628 \\
\hline$\geq 60$ years & 96 & $7.33 \pm 2.47$ & & \\
\hline$<60$ years & 88 & $7.50 \pm 2.26$ & & \\
\hline Gender & & & 0.266 & 0.791 \\
\hline Male & 126 & $7.38 \pm 2.40$ & & \\
\hline Female & 58 & $7.48 \pm 2.31$ & & \\
\hline Lymphatic metastasis & & & 7.027 & 0.000 \\
\hline Yes & 76 & $6.11 \pm 1.74$ & & \\
\hline No & 108 & $8.32 \pm 2.32$ & & \\
\hline TNM stage & & & 4.281 & 0.000 \\
\hline Stage I/II & 80 & $8.22 \pm 2.34$ & & \\
\hline Stage III & 104 & $6.78 \pm 2.20$ & & \\
\hline $\begin{array}{l}\text { Grade of } \\
\text { differentiation }\end{array}$ & & & 1.509 & 0.133 \\
\hline Middle or low & 60 & $7.03 \pm 2.59$ & & \\
\hline High & 124 & $7.59 \pm 2.24$ & & \\
\hline Tumor diameter & & & 6.420 & 0.000 \\
\hline$\geq 4 \mathrm{~cm}$ & 86 & $6.33 \pm 2.02$ & & \\
\hline$<4 \mathrm{~cm}$ & 98 & $8.36 \pm 2.24$ & & \\
\hline
\end{tabular}

occurrence and development of tumors. Immune cells in the tumor microenvironment can kill tumor cells, but if they are immunosuppressed in the microenvironment, tumor cells can escape. In recent years, studies have demonstrated that DCs are abnormally expressed in tumor tissues, and indeed this may be a potential mechanism of tumor cell immune escape $(15,21,22)$. The current study showed that the percentage of TIDCs, MHC-II positive DCs, and CD54 positive DCs in esophageal cancer decreased, and this was correlated with lymph node metastasis, TNM stage, and lesion size. The density of the TIDCs, the percentage of MHC-II positive DCs, and the percentage of CD54 positive DCs were negatively correlated with the percentage of $\mathrm{CD}^{+}{ }^{+} \mathrm{T}$-lymphocytes and positively correlated with the percentage of $\mathrm{CD}^{+} \mathrm{T}$-lymphocytes $(\mathrm{P}<0.05)$.

When the number of TIDCs are reduced in the tissue, 
Table 6 The correlation of TIDC expression and phenotypes with T-lymphocyte subsets in esophageal cancer tissues

\begin{tabular}{lcc}
\hline Category & $\begin{array}{c}\text { CD4 } \\
\text { TID-lymphocytes } \\
(\%)\end{array}$ & $\begin{array}{c}\mathrm{CD}^{+} \text {T-lymphocytes } \\
(\%)\end{array}$ \\
r value & -0.264 & 0.262 \\
P value & 0.002 & 0.003 \\
MHC-Il positive DCs (\%) & \\
r value & -0.268 & 0.302 \\
P value & 0.001 & 0.000 \\
CD54 positive DCs (\%) & & \\
r value & -0.301 & 0.289 \\
P value & 0.000 & 0.000 \\
\hline
\end{tabular}

TIDCs, tumor infiltrating dendritic cells; MHC-II, major histocompatibility complex-II; DCs, dendritic cells.

the body cannot effectively recognize the tumor cell antigen, resulting in insufficient T-lymphocyte subset proliferation and activation. This results in ineffective inhibition of tumor angiogenesis and ineffective recruitment of effector T-lymphocytes in the tumor tissue, hindering the body's ability to kill esophageal cancer cells. In addition, under special circumstances, the TIDCs cannot effectively stimulate T-lymphocyte activation, and in fact, exerts an immunosuppressive effect. This is largely due to the immature or abnormal differentiation of the TIDCs, which is characterized by low positive rates of MHC-II and CD54 $(23,24)$. In the tumor microenvironment, immature DC cells have strong antigen uptake and processing ability, but they cannot activate $\mathrm{T}$ cells due to the lack of a variety of costimulatory molecules, resulting in the incompetence or low response of $\mathrm{T}$ cells. Our study demonstrated a decreased number of TIDCs in esophageal cancer tissues and most of the DCs were functionally immature. These immature DCs inhibit the proliferation of effector T-lymphocytes, promote the drift of Th1/Th2 cells to the Th2 phenotype, and finally lead to the rapid growth and metastasis of esophageal cancer cells.

This report also revealed that the decrease in the percentage of TIDCs, MHC-II positive DCs, and CD54 positive DCs in esophageal cancer tissue is related to lymph node metastasis, TNM stage, and lesion size in patients with esophageal cancer. The small number of TIDCs and their immature function in esophageal cancer tissue are conducive to the rapid growth of esophageal cancer cells.
In addition, this study showed that the density of TIDCs, MHC-II positive DCs, and CD54 positive DCs were significantly negatively correlated with $\mathrm{CD}^{+}{ }^{+} \mathrm{T}$-lymphocytes and positively correlated with $\mathrm{CD} 8^{+} \mathrm{T}$-lymphocytes $(\mathrm{P}<0.05)$. Stimulated by anti-inflammatory factors, $\mathrm{CD}^{+}$ T-lymphocytes mainly differentiate into regulatory T-lymphocytes, resulting in tumor immune escape. Under the stimulation of inflammatory factors, $\mathrm{CD} 8^{+}$ T-lymphocytes differentiate into cytotoxic T-lymphocytes and kill tumor cells. The increase of $\mathrm{CD}^{+}{ }^{+} \mathrm{T}$-lymphocytes and the decrease of $\mathrm{CD}^{+} \mathrm{T}$-lymphocytes in tumor tissues indicates a Th1/Th2 immunity drift towards Th2 immunity. Previous studies have demonstrated that the drift of Th1/ Th2 immunity to Th2 immunity is an important mechanism of immune escape in various tumor cells $(25,26)$. Thus, small numbers and low functionality of DCs in esophageal cancer tissue can lead to the imbalance of immune function, thereby promoting the growth of tumor cells.

There are several main mechanisms through which DCs exert their anti-tumor activity. First, DCs can exert antitumor activity via high expression of MHC-I and MHCII molecules. MHC molecules recognize tumor antigens and form peptide-MHC molecular complexes, which are presented to T-lymphocytes. This initiates the MHC-I restricted cytotoxic T-lymphocyte reaction and the MHCII restricted $\mathrm{CD}^{+}$Th1 reaction (27). The high expression of costimulatory molecules on DCs also provides a second signal molecule for the activation of T-lymphocytes and initiation of the immune response $(28,29)$. Second, DCs can combine with T-lymphocytes to secrete a large number of inflammatory factors, such as interleukin (IL)-12, to activate T-lymphocyte proliferation, induce cytotoxic $\mathrm{T}$ lymphocyte production, dominate Th1 immune response, and kill tumor cells (30). Third, DCs can secrete specific chemotactic initial T-lymphocytes to promote the aggregation of T-lymphocytes in tumor tissues, enhance the cytotoxic effect of T-lymphocytes on tumor cells, maintain the aggregation of effector T-lymphocytes in tumor tissues, and release IL-12, interferon (IFN)- $\gamma$, and other antiangiogenic substances to inhibit tumor angiogenesis (31). DCs can also directly present antigen peptides to $\mathrm{CD}^{+} \mathrm{T}$-lymphocytes and activate $\mathrm{CD} 8^{+}$T-lymphocytes with the help of activated CD4 ${ }^{+}$T-lymphocytes. CD4 ${ }^{+}$T-lymphocytes and CD8 ${ }^{+}$ T-lymphocytes can further enhance the body's anti-tumor immune response by secreting cytokines or via direct killing. When the number of TIDCs decreases and its function is low, the cytotoxic effect of TIDCs on tumor cells is inhibited, promoting the growth and survival of 
tumor cells.

There were several limitations to this investigation. The study only analyzed the correlation of TIDC density and phenotypes with clinicopathological features of patients. Only lymph node metastasis, high TNM stage, and large lesions were used as indicators of poor prognosis in this study. Indeed, TIDC density and phenotypes may have great predictive value for prognosis in patients with esophageal cancer. Moreover, the short follow-up period in this study did not allow for an in depth exploration of the relationship between TIDC density and phenotypes and postoperative recurrence, metastasis, and mortality. Finally, we did not detect vascular endothelial growth factor in esophageal cancer.

In conclusion, this report demonstrated low expression and function of TIDCs in patients with esophageal cancer, and this was related to the imbalance of T-lymphocyte subsets, lymph node metastasis, TNM stage, and lesion size.

\section{Acknowledgments}

Funding: None.

\section{Footnote}

Reporting Checklist: The authors have completed the MDAR reporting checklist. Available at https://dx.doi. org/10.21037/jgo-21-578

Data Sharing Statement: Available at https://dx.doi. org/10.21037/jgo-21-578

Conflicts of Interest: All authors have completed the ICMJE uniform disclosure form (available at https://dx.doi. org/10.21037/jgo-21-578). The authors have no conflicts of interest to declare.

Ethical Statement: The authors are accountable for all aspects of the work in ensuring that questions related to the accuracy or integrity of any part of the work are appropriately investigated and resolved. All procedures performed in this study involving human participants were in accordance with the Declaration of Helsinki (as revised in 2013). The study was approved by the ethics committee of the First Affiliated Hospital of Jiamusi University (Ethics Approval No.: 2019-01-23) and informed consent was taken from all the patients.

Open Access Statement: This is an Open Access article distributed in accordance with the Creative Commons Attribution-NonCommercial-NoDerivs 4.0 International License (CC BY-NC-ND 4.0), which permits the noncommercial replication and distribution of the article with the strict proviso that no changes or edits are made and the original work is properly cited (including links to both the formal publication through the relevant DOI and the license). See: https://creativecommons.org/licenses/by-nc-nd/4.0/.

\section{References}

1. Nilsson K, Klevebro F, Rouvelas I, et al. Surgical morbidity and mortality from the multicenter randomized controlled NeoRes II trial: standard versus prolonged time to surgery after neoadjuvant chemoradiotherapy for esophageal cancer. Ann Surg 2020;272:684-9.

2. Fan J, Liu Z, Mao X, et al. Global trends in the incidence and mortality of esophageal cancer from 1990 to 2017. Cancer Med 2020;9:6875-87.

3. Xi Y, Dong W, Qiao L, et al. Trends in incidence and mortality of esophageal cancer in Inner Mongolia, 20102015. Thorac Cancer 2020;11:2440-8.

4. Hong M, Jiang Z, Zhou YF. Effects of thermotherapy on Th1/Th2 cells in esophageal cancer patients treated with radiotherapy. Asian Pac J Cancer Prev 2014;15:2359-62.

5. Lan F, Xu B, Li J. A low proportion of regulatory T cells before chemoradiotherapy predicts better overall survival in esophageal cancer. Ann Palliat Med 2021;10:2195-202.

6. Li F, Zhou X, Chen M, et al. Regulatory effect of LncRNA DRAIC/miR-149-5p/NFIB molecular network on autophagy of esophageal cancer cells and its biological behavior. Exp Mol Pathol 2020;116:104491.

7. Taghvaei F, Rastin SJ, Milani AT, et al. Carboplatin and epigallocatechin-3-gallate synergistically induce cytotoxic effects in esophageal cancer cells. Res Pharm Sci 2021;16:240-9.

8. Nakhaeepour Z, Mashreghi M, Matin MM, et al. Multifunctional $\mathrm{CuO}$ nanoparticles with cytotoxic effects on KYSE30 esophageal cancer cells, antimicrobial and heavy metal sensing activities. Life Sci 2019;234:116758.

9. Wi TI, Byeon Y, Won JE, et al. Selective tumor-specific antigen delivery to dendritic cells using mannose-labeled poly(d, l-lactide-co-glycolide) nanoparticles for cancer immunotherapy. J Biomed Nanotechnol 2020;16:201-11.

10. Matsumoto M, Takeda Y, Seya T. Targeting Toll-like receptor 3 in dendritic cells for cancer immunotherapy. Expert Opin Biol Ther 2020;20:937-46.

11. Mayoux M, Roller A, Pulko V, et al. Dendritic cells dictate 
responses to PD-L1 blockade cancer immunotherapy. Sci Transl Med 2020;12:eaav7431.

12. Audsley KM, McDonnell AM, Waithman J. Crosspresenting XCR1+ dendritic cells as targets for cancer immunotherapy. Cells 2020;9:565.

13. Zhang M, Shi Y, Zhang Y, et al. miRNA-5119 regulates immune checkpoints in dendritic cells to enhance breast cancer immunotherapy. Cancer Immunol Immunother 2020;69:951-67.

14. Han N, Li X, Wang Y, et al. Increased tumor-infiltrating plasmacytoid dendritic cells promote cancer cell proliferation and invasion via $\mathrm{TNF}-\alpha / \mathrm{NF}-\kappa \mathrm{B} / \mathrm{CXCR}-$ 4 pathway in oral squamous cell carcinoma. J Cancer 2021;12:3045-56.

15. Kießler M, Plesca I, Sommer U, et al. Tumor-infiltrating plasmacytoid dendritic cells are associated with survival in human colon cancer. J Immunother Cancer 2021;9:e001813.

16. Liu J, Li HJ, Luo YL, et al. Programmable delivery of immune adjuvant to tumor-infiltrating dendritic cells for cancer immunotherapy. Nano Lett 2020;20:4882-9.

17. Jiang L, Fang X, Wang H, et al. Ovarian cancer-intrinsic fatty acid synthase prevents anti-tumor immunity by disrupting tumor-infiltrating dendritic cells. Front Immunol 2018;9:2927.

18. Yang W, Yu J. Immunologic function of dendritic cells in esophageal cancer. Dig Dis Sci 2008;53:1739-46.

19. Liu Y, Mu Y, Zhang A, et al. Cytokine-induced killer cells/dendritic cells and cytokine-induced killer cells immunotherapy for the treatment of esophageal cancer in China: a meta-analysis. Onco Targets Ther 2017;10:1897-908.

20. Yuan X, Zhang AZ, Ren YL, et al. Cytokine-induced killer cells/dendritic cells and cytokine-induced killer cells immunotherapy for the treatment of esophageal cancer: a meta-analysis. Medicine (Baltimore) 2021;100:e24519.

21. Tran Janco JM, Lamichhane P, Karyampudi L, et al. Tumor-infiltrating dendritic cells in cancer pathogenesis. J Immunol 2015;194:2985-91.

22. Aleksandrova E, Vlaykova T, Ananiev J, et al. Association of IL-12Bpro polymorphism with tumorinfiltrating dendritic cells in colorectal cancer. J BUON 2017;22:888-93.

23. Yan J, Liu B, Shi Y, et al. Class II MHC-independent suppressive adhesion of dendritic cells by regulatory $\mathrm{T}$ cells in vivo. J Exp Med 2017;214:319-26.

24. Spallanzani RG, Torres NI, Avila DE, et al. Regulatory dendritic cells restrain $\mathrm{NK}$ cell IFN- $\gamma$ production through mechanisms involving NKp46, IL-10, and MHC class I-specific inhibitory receptors. J Immunol 2015;195:2141-8.

25. Zhao X, Liu J, Ge S, et al. Saikosaponin A inhibits breast cancer by regulating Th1/Th2 balance. Front Pharmacol 2019;10:624.

26. Ma J, Liu H, Wang X. Effect of ginseng polysaccharides and dendritic cells on the balance of Th1/Th2 T helper cells in patients with non-small cell lung cancer. J Tradit Chin Med 2014;34:641-5.

27. Takakura K, Koido S, Kan S, et al. Prognostic markers for patient outcome following vaccination with multiple MHC Class I/II-restricted WT1 peptide-pulsed dendritic cells plus chemotherapy for pancreatic cancer. Anticancer Res 2015;35:555-62.

28. Mazorra Z, Lavastida A, Concha-Benavente F, et al. Nimotuzumab induces NK cell activation, cytotoxicity, dendritic cell maturation and expansion of EGFR-specific $\mathrm{T}$ cells in head and neck cancer patients. Front Pharmacol 2017;8:382.

29. Marigo I, Zilio S, Desantis G, et al. T cell cancer therapy requires CD40-CD40L activation of tumor necrosis factor and inducible nitric-oxide-synthase-producing dendritic cells. Cancer Cell 2016;30:651.

30. Correale P, Micheli L, Vecchio MT, et al. A parathyroidhormone-related-protein (PTH-rP)-specific cytotoxic T cell response induced by in vitro stimulation of tumourinfiltrating lymphocytes derived from prostate cancer metastases, with epitope peptide-loaded autologous dendritic cells and low-dose IL-2. Br J Cancer 2001;85:1722-30.

31. Martinet L, Filleron T, Le Guellec S, et al. High endothelial venule blood vessels for tumor-infiltrating lymphocytes are associated with lymphotoxin $\beta$-producing dendritic cells in human breast cancer. J Immunol 2013;191:2001-8.

(English Language Editor: J. Teoh)

Cite this article as: Pei Y, Zhu Y, Wang X, Xu L. The expression and clinical value of tumor infiltrating dendritic cells in tumor tissues of patients with esophageal cancer. J Gastrointest Oncol 2021;12(5):1996-2003. doi: 10.21037/jgo-21578 